\title{
The operational difficulty of standardizing frequency restoration products
}

\section{Conference Paper}

\section{Author(s):}

Scherer, Marc; Pfister, Michael; Hug, Gabriela

Publication date:

2017

Permanent link:

https://doi.org/10.3929/ethz-b-000231949

Rights / license:

In Copyright - Non-Commercial Use Permitted

Originally published in:

https://doi.org/10.1109/EEM.2017.7981952 


\section{The Operational Difficulty of Standardizing Frequency Restoration Products}

\author{
Marc Scherer \\ Swisscom Energy Solutions Ltd \\ Switzerland \\ marc.scherer@swisscom.com
}

\author{
Michael Pfister \\ ETH Zürich \\ Switzerland \\ micpfist@ee.ethz.ch
}

\author{
Gabriela Hug \\ ETH Zürich \\ Switzerland \\ hug@eeh.ee.ethz.ch
}

\begin{abstract}
This paper assesses operational impacts of the Continental European power plant portfolio on a harmonization of load-frequency control. The technical basics are presented together with an 18-area system of Continental Europe implemented in SIMULINK. The results reveal significant differences in frequency restoration among control areas. These differences originate from country-specific power plant portfolios as well as non-standardized control and activation parameters. An evaluation of pro rata and merit order activation demonstrates that frequency restoration can be harmonized to a certain extent but can cause both improvement or deterioration for control areas. Product standardization in the scope of market design and the choice of technical parameters in the scope of system operation are inextricably linked.
\end{abstract}

Index Terms-Automatic generation control, frequency control, frequency restoration reserves, reserve activation.

\section{INTRODUCTION}

Nowadays, national system operators are responsible for frequency control either as Independent System Operators (ISOs), which operate only, or as Transmission System Operators (TSOs), which operate and own the transmission system. Active power reserves for frequency restoration are assessed and dimensioned in line with international standards and minimum requirements, but the relevant economic and technical characteristics are stipulated by national products, implementations, and regulatory authorities [1]. This complicates standardizing products for frequency control purposes across countries, in particular in the scope of the EC's Third Energy Package and its "Network Codes" and "Guidelines" [2].

So far, several studies have been carried out comparing regional ancillary service markets as well as their reserve deployment and control structures [3]-[6]. An overview of European control parameters is given in [7] and a discussion of different activation schemes for Automatic Generation Control (AGC) in [8]. Reduced power systems for capturing frequency control and market participant behavior showed good performance and results of high practical relevance: Active power reserve dimensioning was analyzed across Europe in [9] and the dependency of market activity and frequency quality was modeled in [10].

Our focus is on modeling the impact of harmonized reserve activation parameters for the Continental European power system. In this context, the contribution of this paper is twofold:

978-1-5090-5499-2/17/\$31.00 (c) 2017 IEEE
Assessing the impact of regional production portfolios on frequency restoration and quantifying the effect of harmonized control and activation parameters. We rely on a reduced power system model which captures the frequency control structure and reserve activation logic of Continental Europe. The model is matched to the current decentralized frequency control structure, which allows investigating the technical impact of both different frequency restoration products and changes in the degree of centralization of the control structure.

This paper is organized as follows: Section II elaborates the frequency control basics for investigating the impact of power plant dynamics on frequency restoration including pro rata and merit order activation schemes. Section III presents the simulations and results for Continental Europe. Section IV discusses the findings and the policy implications on frequency performance and product design. Finally, Section V is devoted to conclusions and perspectives.

\section{Methods And Modeling}

\section{A. Technical Basics of Frequency Restoration Products}

In most interconnected power systems, frequency control is a three-tiered approach which involves frequency containment ("primary control"), frequency restoration ("secondary control"), and reserve replacement ("tertiary control"). The associated capacities are referred to as active power reserves or control reserves.

Frequency Containment Reserves (FCR) are the joint responsibility for frequency-response distributed among all TSOs in a synchronous area. Frequency Restoration Reserves (FRR) imply a local responsibility of each TSO for the imbalance in its control area. In Continental Europe, automatic frequency restoration is performed by $\mathrm{AGC}$ also referred to as Load-Frequency Control (LFC). The AGC principles are based on the fact that the quasi-steady state frequency is the same in the entire synchronous area; therefore, decentralized feedback implemented by each control area for responding to the local imbalance contributes to the overall balance in the system. The Area Control Error $A C E$ is the sum of the weighted frequency deviation and the deviations of the net tie-line flow between the control area and its neighbors. The $A C E$ is the control error for a PI controller with anti-windup; its control signal $P^{\mathrm{AGC}}$ is sent to reserve providing units, which deploy the respective amount of active power reserves, i.e. automatic 
FRR. If the frequency bias factor $K_{\mathrm{j}}$ is chosen appropriately, a control area will only compensate for its imbalance, and it will neither counteract its frequency containment contribution nor its share of self-regulation; vice versa, AGC will compensate for a non-delivery of FCR in the respective area. This principle is referred to as non-interactive control [1], [11].

The technical activation logic for FRR can be chosen without regard to the market structure and pricing. In practice, however, automatic FRR are usually activated based on the energy price. For pay-as-bid offers, the units are activated according to merit order. In case of uniform pricing, all units are proportionally activated in parallel, i.e. pro rata. As the AGC control signal is typically sent to the $n$ providers as a contribution factor $g_{i}$ relative to their total offer, i.e. $g_{i} \in[0,1]$, let $g=\left(g_{i}\right)$ be a vector of dimension $n$ whose elements correspond to the $n$ provider such that

$$
g_{i}^{\mathrm{mol}}= \begin{cases}\min \left\{\frac{P^{\mathrm{AGC}}-\sum_{j=1}^{i-1} P_{j}^{\mathrm{FRR}}}{P_{i}^{\mathrm{FRR}}}, 1\right\} & \text { if } \sum_{j=1}^{i-1} P_{j}^{\mathrm{FRR}}<P^{\mathrm{AGC}}, \\ 0 & \text { otherwise }\end{cases}
$$

for a merit order activation logic, and

$$
g_{i}^{\mathrm{pro}}=\min \left\{\frac{P_{i}^{\mathrm{AGC}}}{\sum_{i=1}^{n} P_{i}^{\mathrm{FRR}}}, 1\right\}
$$

for a pro rata activation logic, where $P_{i}^{\mathrm{FRR}}$ is the total amount of contracted reserves of provider $i$. Nowadays, most countries have implemented a pro rata activation [12]. Note that the choice of activation logic does not add additional dynamics. Let $H^{\mathrm{FRR}}(s)$ be the transfer function for each reserve provider, and the total response is given by

$$
\begin{aligned}
\sum_{i=1}^{n} g_{i}^{\mathrm{mol}} P_{i}^{\mathrm{FRR}} H^{\mathrm{FRR}}(s) & =P^{\mathrm{AGC}} H^{\mathrm{FRR}}(s) \\
& =\sum_{i=1}^{n} g_{i}^{\mathrm{pro}} P_{i}^{\mathrm{FRR}} H^{\mathrm{FRR}}(s) .
\end{aligned}
$$

Therefore, both activation logics lead to the same response in case of a common linear time-invariant behavior. For different non-linear characteristics which usually apply to reallife power plants such as ramping restrictions and response delays, Formula 3 does not hold.

\section{B. Modeling of European Frequency Control Structures}

The model used in this paper comprises control structure and components required for FCR and automatic FRR. Figure 1 shows the model. Figure 1a shows a decentralized frequency control structure configuration. Each area contains FCR and FRR power plants, whereas load self-regulation is considered for the whole synchronous area. Since short-term phenomena such as power plant dynamics and small-signal frequency swings are not significant for AGC, the modeled power system shares one common frequency, i.e. the system

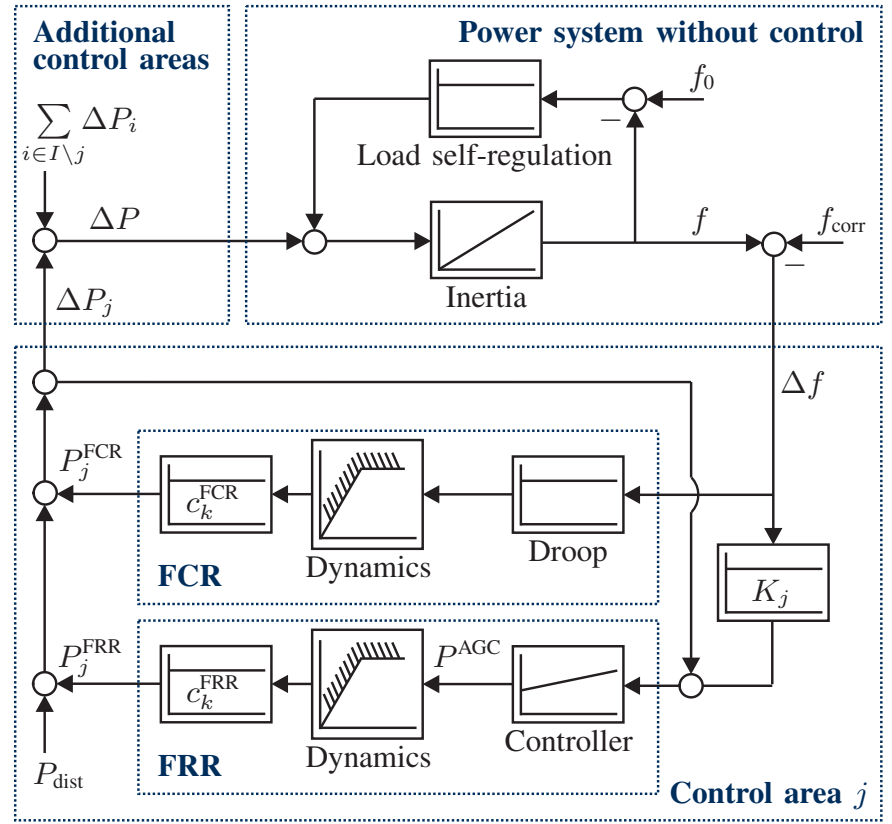

(a) Decentralized frequency control structure.

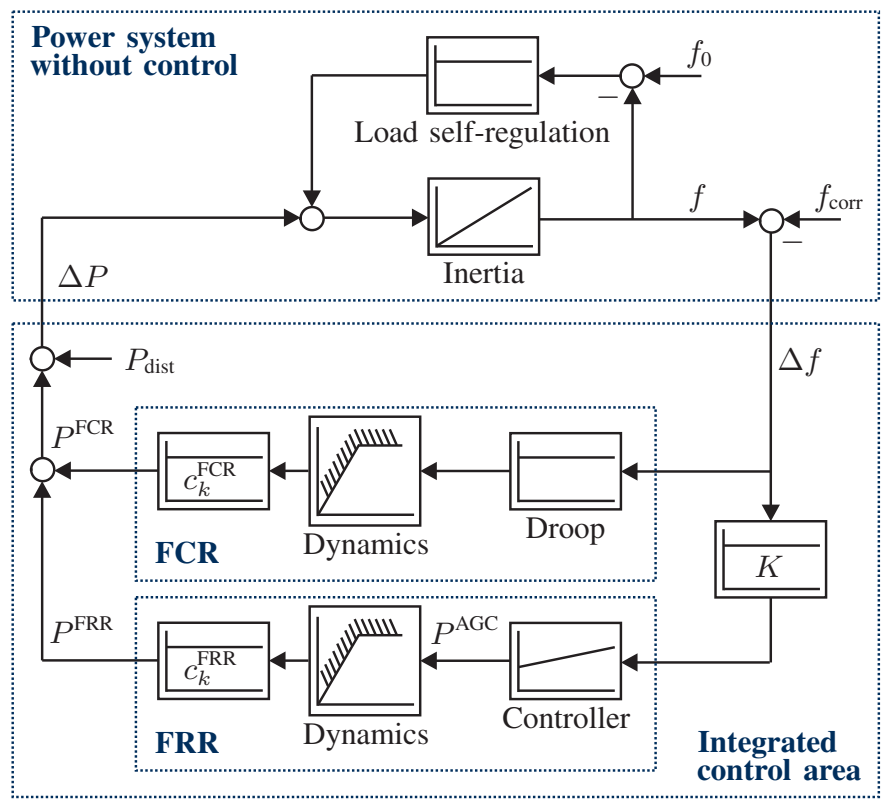

(b) Centralized frequency control structure.

Fig. 1: Overview of the dynamic frequency model used for frequency restoration analyses.

frequency deviation is a result of the one common system inertia block. Load self-regulation and inertia would determine the system frequency without additional active power reserves, i.e. without FCR and FRR. The decentralized setup considers 18 countries that are part of ENTSO-E's Regional Group Continental Europe (RGCE).

Figure $1 \mathrm{~b}$ shows a centralized configuration, where one control area comprises the whole synchronous area. AGC is carried out by one central controller implying one integrated 


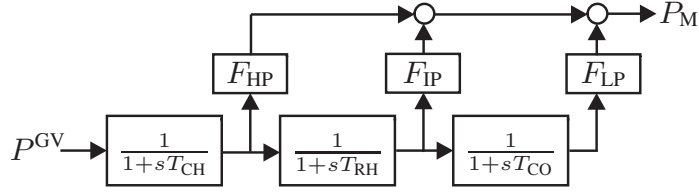

(a) Tandem-compound single reheat.

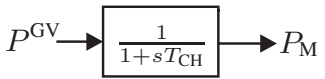

(b) Non-reheat.

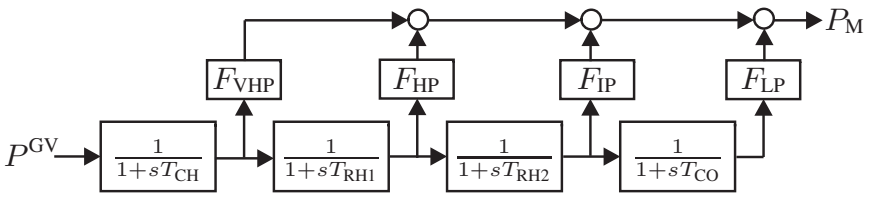

(c) Tandem-compound double-reheat.

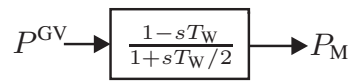

(d) Hydro.

Fig. 2: Overview of the turbine models.

frequency control area. This setup serves as a reference to evaluate the impact of an integrated one-area approach.

The dynamics in the FCR and FRR loops include transfer functions for different types of turbines, governor systems and generating units (see Section II-C). The shares $c_{\mathrm{FCR}}$ and $c_{\mathrm{FRR}}$ of different types of power plants can change with seasonality and the auctioning in the ancillary service markets; therefore, a typical mix is used in order to grant comparability and obtain realistic (default) values without precisely knowing the unit allocation.

Manual reserves, such as manual FRR or replacement reserves, are not explicitly considered; they can either be activated reactively (after the occurrence of an imbalance) or proactively (predicting deterministic imbalances) [13]. They do not change AGC dynamics and can be assumed to be implicitly included in the unit commitment.

\section{Production Portfolio Dynamics}

Simulations dealing with restoration scenarios in power systems require detailed models including non-linearities, while in stability scenarios much simpler models of power plants, loads and control are sufficient to examine global behavior of FCR and FRR in interconnected power systems [14].

The frequency model considers five different power plant technologies: Combined Cycle Gas Turbine (CCGT), gas, coal, nuclear and hydro. Today, the share of inverter-based active power reserve providers is negligibly small [7]. Each dynamic model consists of a governor transfer function and a turbine block-diagram [15]. The models are summarized in Figure 2.

Governors determine and react to frequency deviations in the power system by adjusting the turbine inflow. The inflow can be steam, gas or water. The governor outputs a power $P_{\mathrm{V}}$ which is passed on to the turbine. If the system frequency is below nominal frequency, mechanical torque of the turbine is increased and vice versa. For steam and gas turbine systems, a mechanical-hydraulic speed-governing system is used, whereas for hydro turbines a governing system with transient droop compensation addresses the non-minimal phase behavior of hydro turbines [15].

The five block diagrams in Figure 2 represent the turbines for CCGT (tandem compound single reheat), gas (non-reheat), coal and nuclear (tandem compound double-reheat) and hydro. A turbine unit converts the inflow $P_{\mathrm{GV}}$ into mechanical power $P_{\mathrm{m}}$. For CCGT, time delays are introduced due to steam chest and inlet piping $\left(T_{\mathrm{CH}}\right)$, re-heaters $\left(T_{\mathrm{RH}}\right)$ and crossover piping $\left(T_{\mathrm{CO}}\right)$. A valve controls the inlet to the HighPressure (HP), Intermediate-Pressure (IP) and Low-Pressure (LP) turbine fraction. The gas power plant is modeled with a fast non-reheat turbine. For coal and nuclear power plants, a tandem-compound double-reheat turbine system is used with four turbine fractions. A valve controls the inlet to a Very High-Pressure (VHP) turbine followed by a HP, IP, and LP turbine. Time delays are due to steam chest and inlet piping $\left(T_{\mathrm{CH}}\right)$, re-heaters $\left(T_{\mathrm{RH}}\right)$ and cross-over piping $\left(T_{\mathrm{CO}}\right)$.

For nuclear power plants, higher delays are used than for coal power plant dynamics. The characteristics of hydro turbines are determined by the dynamics of water flow in the penstock [15]. In addition to governor and turbine dynamics, typical ramp rates are incorporated in the modeling of the power plants [7].

\section{Model Parametrization and Limitations}

Different scenarios can be considered when modeling frequency imbalances. In the RGCE, peak and low load amounts to $300 \mathrm{GW}$ and $150 \mathrm{GW}$, respectively. The simulations in this paper are based on a low load scenario and system parameters given in [16]. For each area, the available FCR and FRR is considered. In all dynamic models, an AGC activation delay of $45 \mathrm{~s}$ is incorporated which represents an average time elapsed between frequency deviation detection and AGC activation.

Depending on the type, technology, and manufacturer of a unit, the dynamics of units providing ancillary services can differ vastly. They cannot be fully captured with a reduced power system model. The same applies to the AGC implementation: The providers are assumed to directly react to $P_{\mathrm{AGC}}$ (see Section II-A), but in practice, the AGC signal from the TSO can also be routed or split by the provider to multiple units; the precise handling is often up to the ancillary service provider and only limited by TSO-specific requirements tested during pre-qualification [17], i.e. the calibration of the AGC loop leaves several degrees of freedom to the providers (see the discussion in Section IV).

\section{Simulations And Results}

In the following, incidents are investigated with different activation logics. The difference is made between small imbalances representing normal operation and large imbalances that require the TSO to activate all the available FRR, i.e. full reserve activation. With the latter, the technical capabilities 
of today's AGC implementation can be investigated without assessing the actual reserve dimensioning, as situations where the needed amount is higher than the available amount of reserves inherently lead to a (steady-state) frequency deviation (independent of AGC parameters). Moreover, the technical frequency restoration capabilities with a decentralized frequency control structure are compared to a centralized frequency control structure.

Note that it is assumed that an imbalance only occurs in one area at the same time, i.e. when comparing different countries, only one imbalance in one area occurs. Therefore, the different frequency plots represent scenarios which are independent of each other.

\section{A. Small Imbalances in Normal Operation}

Each control area in the RGCE has the capacity to balance a loss of $100 \mathrm{MW}$, which stands for a small imbalance that can occur during normal operation. For example, the control block comprising Serbia, Montenegro, and Macedonia has a small amount of automatic FRR available, whereas Germany procures the largest amount in the RGCE [7].

In Figure 3, a loss of $100 \mathrm{MW}$ is simulated after $10 \mathrm{~s}$ with a decentralized frequency control structure for Portugal, Poland, Germany as well as Serbia, Montenegro, and Macedonia. Since FCR are inherently shared reserves, all four scenarios show the same frequency trajectory until FRR is activated. For all four control areas, FRR is activated pro rata in all areas for the sake of comparability. Even though Germany has by far the largest amount of reserves, Portugal is fastest in restoring frequency. The power plant portfolio dynamics have a stronger impact than the number of bids that get activated. As power plant and control structure dynamics are incorporated in the model, the control area with the highest amount of reserves is not the fastest although all providing units are activated with a pro rata logic. Due to a large amount of reserves dominated by coal power plants, which have strong ramping restrictions, Poland has a significantly slower response. Portugal on the other hand has a significant share of hydro and gas power, and fast AGC parameters.

Significant differences in frequency restoration times exist among control areas in Continental Europe. The simulation shows that fast countries such as Portugal restore a loss of $100 \mathrm{MW}$ below one minute while the slow areas such as Poland need more than 10 minutes. Note that such small imbalances do not significantly affect system frequency. Typically, small imbalances cause frequency deviations that stay within the regular frequency dead band, which is also used for time correction (see $f_{\text {corr }}$ in Figure 1).

\section{B. Full Reserve Activation}

In Figure 4, the same countries as in Section III-A are considered with a decentralized frequency control structure. The reference incident is set equal to the net amount of local reserves available in an area, i.e. the imbalance simulated in all four control areas equals to a loss of the amount of their full FRR. All areas activate their reserves with a pro rata logic. It

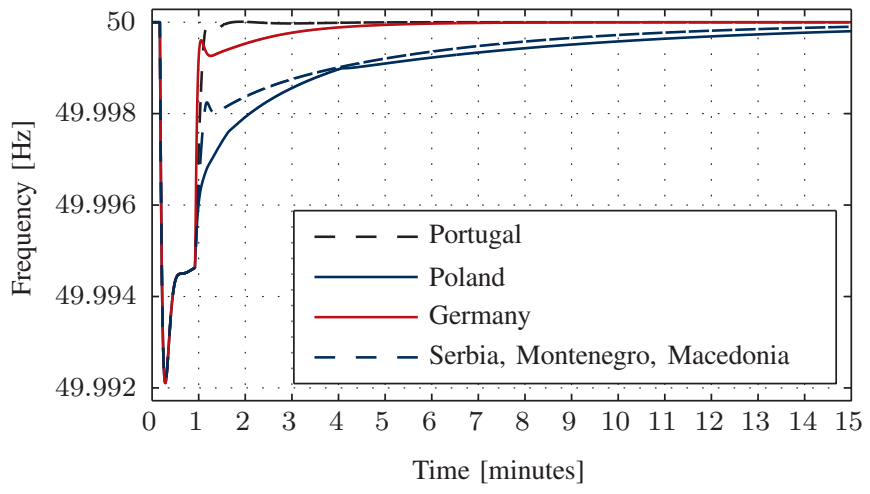

Fig. 3: Frequency responses for a loss of $100 \mathrm{MW}$ representing "normal operation" in different control areas.

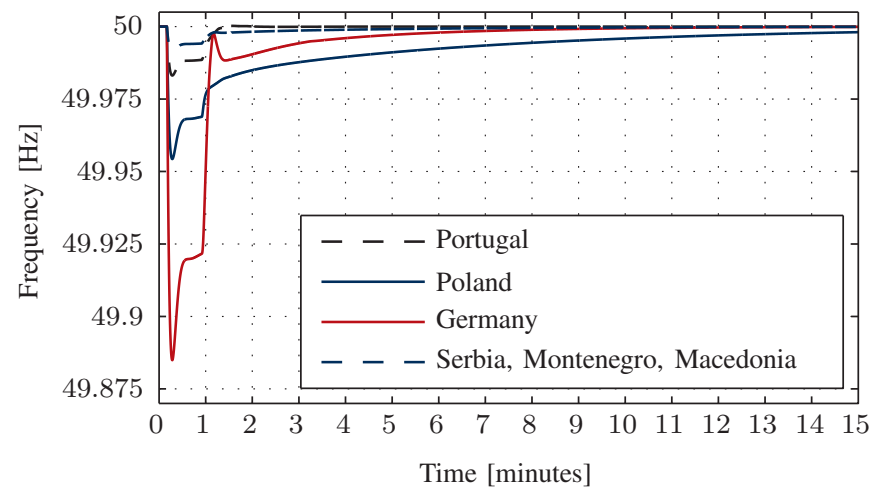

Fig. 4: Frequency restoration for full AGC activation in different countries.

is noteworthy that only in Germany the amount of automatic FRR exceeds the nominal power of their largest power plant.

The fastest and slowest performances do not significantly differ. In the case of full reserve activation, the fastest country to restore nominal frequency to $5 \%$ of its initial deviation is Portugal with a bit more than one minute. Poland needs around 14 minutes.

\section{Harmonization}

In Figure 5, the average frequency restoration trajectory of all 18 areas for a loss of $100 \mathrm{MW}$ in the decentralized frequency control structure is plotted. The response is simulated for a pro rata and a merit order activation logic. Both logics activate according to a bid size of $10 \mathrm{MW}$. The merit order list for a merit order activation is simplified, i.e. static and always the same with hydro being the cheapest.

In the same manner as FCR, automatic FRR are shared among all control areas in a spirit of solidarity in the centralized structure. The FRR of all countries are bundled in one AGC loop and activated with a pro rata or merit order activation logic. With a pro rata activation, all providing units receive the AGC signal in parallel. This creates a fast frequency response. With merit order, only the cheapest FRR bids are activated to fully compensate the imbalance. For the 


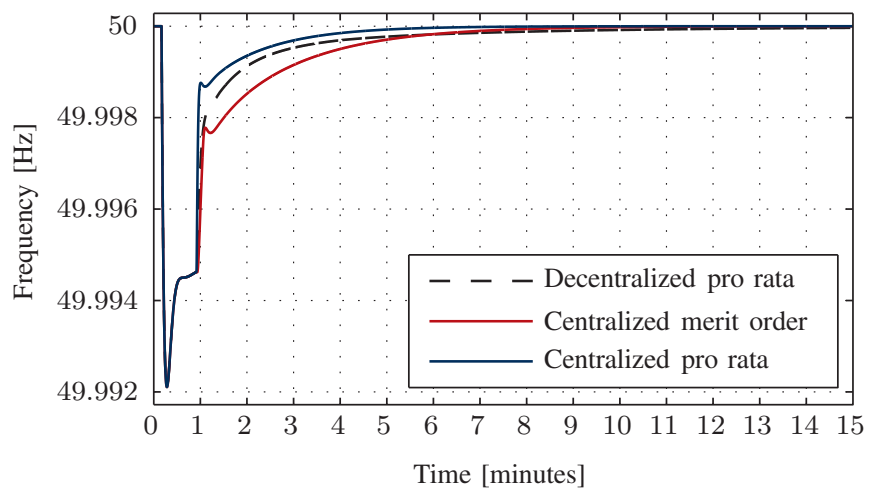

Fig. 5: Comparison of a centralized frequency restoration approach and the average frequency response of the 18 countries with a decentralized frequency control structure.

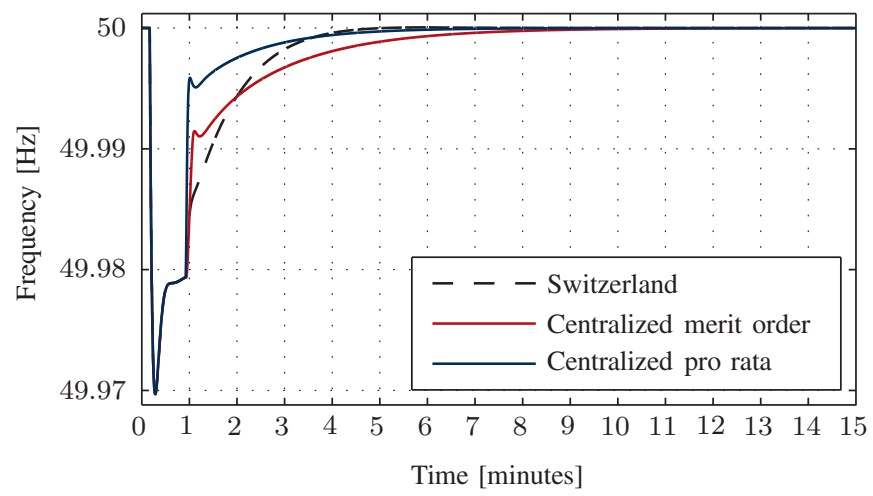

Fig. 6: Comparison in frequency restoration of centralized with decentralized frequency control structure.

sake of comparability, the AGC parameters are not changed for the different activation logics.

As can be seen in Figure 5, the centralized pro rata automatic FRR activation restores a $100 \mathrm{MW}$ imbalance faster than the decentralized structure. Simulation shows that ten control areas restore frequency faster with the centralized pro rata than with the decentralized pro rata structure. Only three control areas restore a $100 \mathrm{MW}$ imbalance slower than the centralized merit order activation scheme.

In Figure 6, the response of Switzerland with a decentralized frequency control structure for a full AGC activation incident is compared to the centralized frequency restoration process. While the centralized pro rata scheme restores frequency faster than the decentralized pro rata activation of Switzerland, the merit order activation takes longer to restore frequency.

A comparison between the 18 control areas' decentralized frequency restoration times and a centralized control structure shows that for a full reserve activation, the centralized pro rata scheme is faster for 14 control areas than the decentralized scheme. The centralized merit order activation scheme is faster for seven control areas. Summing up, more control areas profit from a centralized control structure in case of large imbalances than for small imbalances.
For the centralized pro rata and merit order structure the standard bid size is changed from $10 \mathrm{MW}$ to $30 \mathrm{MW}$ to obtain a reasonable activation share also for small reserve demands. Simulation shows that this change does not affect the restoration time of the pro rata scheme but slows down the merit order frequency restoration by more than two minutes for the small $100 \mathrm{MW}$ imbalances. For large imbalances, the bid size change slows down the centralized merit order structure by less than $10 \mathrm{~s}$.

\section{Policy IMPLiCATION}

The main findings of the different frequency restoration implementations in this work are as follows:

- Significant differences in frequency restoration exist among control areas. Simulations show that fast areas restore imbalances within a minute while slow areas need up to 15 minutes.

- Power plant dynamics influence frequency restoration, and controller parameters impact the frequency restoration times. For example, areas with lots of hydro power exhibit a higher integral gain than control areas with lots of thermal power plants.

- Bid sizes can affect the activation performance depending on the activation scheme. The smaller the pro rata bids, the more bids get activated in parallel and the faster the frequency restoration. However, with a centralized control structure, a pro rata activation may become rather impractical for activating small amounts of reserves (noise, filtering, monitoring).

- A one-area setup harmonizes frequency restoration times for small and large imbalances among countries. Pro rata activation is slower than merit order activation for countries which feature a high integral gain today. A merit order activation logic deteriorates frequency restoration for the majority of countries.

- For small imbalances, the FRR parameters have a minor impact on frequency quality, as the frequency stays in a narrow range in any case. For large imbalances, such as market-induced imbalances [10], the parameters can significantly affect frequency quality.

The discussion of product standardization is usually limited to deployment characteristics (ramping, full activation time, activation duration) of active power reserve products; however, the technical activation characteristics, i.e. AGC parameters, have a significant impact on the reserve response and the resulting frequency quality. The values used today have evolved over time: The historical conditions have substantially determined the production portfolio, and TSOs have chosen the AGC parameters accordingly.

Technical AGC parameters need to be considered in the discussion of standard products in respect of the technical capability of local reserve providers and the targeted global frequency quality. The AGC signal should only be locally limited to match the providing unit (for example, filtering to avoid power oscillations) in order not to anticipate performance limitations. A clear distinction should be made between technical 
minimum requirements, such as given in [1], [16], or [17], and the target performance of standard products. Minimum requirements cannot necessarily be used as design criteria: If they are set equal, chances are high that providers capable of providing fast frequency restoration will unnecessarily limit their technical capability. Instead, to incentivize performance, the product design should differentiate the technical capability of the provider, for example, by a pay as performance-based remuneration. This would require that the energy price for the delivered active power reserves is a function of the physical response in power and not only a function of the net energy per schedule period.

\section{Closing Remarks}

\section{A. Conclusion}

This paper analyzed and discussed the impact of generation portfolios and control parameters on frequency restoration. Although the European legislation, driven in particular by the vision of an integrated pan-European market, is pushing towards a consolidation of the European electricity sector, the frequency control structure for transmission system operation has mostly been kept in its decentralized form, where national TSOs manage frequency control decentrally. In this context, we showed the impact of changing the AGC activation logic and the degree of centralization.

Regional differences in the production portfolio and the technical AGC implementations make it difficult to standardize products for frequency restoration ensuring a harmonized control performance. Theoretically, AGC parameters would need to be adjusted in real-time in accordance with ancillary service market activity and the providers' unit commitment. It seems more practical to consider the technical capability in the definition of standard products; a pay as performance-based remuneration for the deployed reserve energy could address this issue with a market-based solution. This implies that wellestablished technical minimum requirements, which have been defined for conventional power plants, should not directly be transformed into target performance parameters of standard products. Instead, the product design should make use of the individual technical capability of ancillary service providers. Product standardization in the scope of market design and the choice of technical parameters in the scope of system operation are inextricably linked.

\section{B. Outlook}

The results of this paper are solely related to technical parameters. Further investigation is required to make statements about economic aspects such as total costs. Moreover, this work limits the provision of reserves to conventional power plants. For future scenarios, larger shares of inverter-based generators and aggregated loads providing reserves should be considered. For better grounded statements about potential improvement or deterioration in frequency restoration times, modeling with historical data could be considered.

In this paper, the consideration of a centralized control structure across Continental Europe only served as a reference to compare different activation logics of AGC resources; however, a consolidation of control areas to access synergy effects is an obvious technical solution to counter the potentially increasing demand for active power reserves. The European work in progress "Guidelines" and "Network Codes" support such a step, which is underlined by concepts such as imbalance netting and common merit order lists. In this context, the impact on and influence of the production portfolio and AGC parameters should be thoroughly considered for future ancillary service products.

\section{REFERENCES}

[1] OpHB-Team, "Policy 1: Load-Frequency Control and Performance," in UCTE Operation Handbook, March 2009.

[2] European Commission, "Directive 2009/28/EC of the European Parliament and of the Council of 23 April 2009 on the Promotion of the Use of Energy from Renewable Sources and Amending and Subsequently Repealing Directives 2001/77/EC and 2003/30/EC," Official Journal of the European Union, vol. 52(L140), pp. 16-32, June 2009.

[3] E. Rivero, J. Barquin, and L. Rouco, "European Balancing Markets," in $8^{\text {th }}$ International Conference on the European Energy Market (EEM), May 2011, pp. 333-338.

[4] J. Frunt, W. Kling, R. Hermans, F. Nobel, and W. de Boer, "Impact of Design Variables on Balancing Markets," in $7^{\text {th }}$ International Conference on the European Energy Market (EEM), June 2010, pp. 1-6.

[5] Tractebel Engineering and Katholieke Universiteit Leuven, "Study of the Interactions and Dependencies of Balancing Markets, Intraday Trade and Automatically Activated Reserves," TREN /C2/84/2007, February 2009, ec.europa.eu.

[6] R. van der Veen and R. Hakvoort, "Balance Responsibility and Imbalance Settlement in Northern Europe - An Evaluation," in $6^{\text {th }}$ International Conference on the European Energy Market (EEM), May 2009, pp. 1-6.

[7] E-Bridge Consulting GmbH and Institute of Power Systems and Power Economics (IAEW), "Impact of Merit Order Activation of Automatic Frequency Restoration Reserves and Harmonised Full Activation Times," December 2015, www.entsoe.eu.

[8] I. Avramiotis-Falireas, P. Zolotarev, A. Ahmadi-Khatir, and M. Zima, "Analysis and Comparison of Secondary Frequency Control Reserve Activation Rules: Pro-Rata vs. Merit Order," in Power Systems Computation Conference (PSCC), August 2014, pp. 1-7.

[9] M. de la Torre Rodríguez, M. Scherer, D. Whitley, and F. Reyer, "Frequency Containment Reserves Dimensioning and Target Performance in the European Power System," in IEEE PES General Meeting Conference Exposition, July 2014, pp. 1-5.

[10] T. Weißbach and E. Welfonder, "High Frequency Deviations Within the European Power System - Origins and Proposals for Improvement," ELECTRA, vol. 242, pp. 9-15, 2009.

[11] N. Jaleeli, L. S. VanSlyck, D. N. Ewart, L. H. Fink, and A. G. Hoffmann, "Understanding Automatic Generation Control," IEEE Transactions on Power Systems, vol. 7, no. 3, pp. 1106-1122, August 1992.

[12] ČEPS, Elia System Operator S.A., Energinet.dk, Swissgrid Ltd., TenneT TSO B.V., and Tennet TSO GmbH, "Comparison of System Balancing Management for Six Electricity Transmission System Operators," GEN and E-Bridge Consulting GmbH, Tech. Rep., September 2013.

[13] F. Abbaspourtorbati, M. Scherer, A. Ulbig, and G. Andersson, "Towards an Optimal Activation Pattern of Tertiary Control Reserves in the Power System of Switzerland," in American Control Conference (ACC), June 2012, pp. 3629-3636

[14] H. Weber, M. Hladky, T. Haase, S. Spreng, and C. Moser, "High Quality Modelling of Hydro Power Plants for Restoration Studies," IFAC Proceedings Volumes, vol. 35, no. 1, pp. 263-268, 2002.

[15] IEEE Committee Report, "Dynamic Models for Steam and Hydro Turbines in Power System Studies," IEEE Transactions on Power Apparatus and Systems, vol. PAS-92, no. 6, pp. 1904-1915, November 1973.

[16] OpHB-Team, "Appendix 1: Load-Frequency Control and Performance," in UCTE Operation Handbook, 2004.

[17] D. Schlipf, M. Scherer, and M. Haller, "Test for Secondary Control Capability," Version 2.1, April 2013, www.swissgrid.ch. 